\title{
APLICAÇÃO DE LIPASES MICROBIANAS NA OBTENÇÃO DE CONCENTRADOS DE ÁCIDOS GRAXOS POLIINSATURADOS
}

Patrícia de Oliveira Carvalho*, Paula Renata Bueno Campos, Maximiliano D’Addio Noffs, Joaquim Gilberto de Oliveira, Mário Tsunezi Shimizu e Daniela Martins da Silva

Curso de Farmácia, Centro de Ciências Biológicas e da Saúde, Universidade São Francisco, Av. São Francisco de Assis, 218, 12916-900 Bragança Paulista - SP

Recebido em 7/12/01; aceito em 11/7/02

\begin{abstract}
APPLICATION OF MICROBIAL LIPASES TO CONCENTRATE POLYUNSATURATED FATTY ACIDS. Several polyunsaturated fatty acids (PUFA) belonging to the ômega 6 series, such as cis-6,9,12 $\gamma$-linolenic acid, as well as those of the ômega 3 series, such as cis-5,8,11,14,17-eicosapentaenoic acid and cis-4,7,10,13,16,19-docosahexaenoic acid are of considerable interest due to their nutritional and therapeutic properties. Methods used for the concentration of PUFA from natural sources include urea adduct formation, solvent winterization, supercritical fluid extraction and lipase-catalyzed reaction. Lipases are known to have little reactivity on PUFA and these acids can be enriched by selective hydrolysis, direct esterification of glycerol with PUFA and interesterification. Since lipase reactions are advantageous with respect to fatty acid, positional specificities and mild incubation condition, these enzymes are considered to be suitable for the production of PUFA concentrates for medical purposes.
\end{abstract}

Keywords: polyunsaturated fatty acid; lipase; fatty acid selectivity.

\section{INTRODUÇÃO}

A importância dos ácidos graxos essenciais (ácido linoléico e ácido $\alpha$-linolênico) na dieta humana foi assunto de grande interesse nas décadas passadas. Estes ácidos que contém duas ou mais insaturações são chamados poliinsaturados e representados por símbolos numéricos, como C18:2 $(9,12)$ que representa o ácido linoléico e o C18:3 $(9,12,15)$, o ácido $\alpha$-linolênico, sendo que o número justaposto ao símbolo $\mathrm{C}$ indica o número de átomos de carbono e o segundo número, a quantidade de duplas ligações. A posição da ligação dupla na cadeia hidrocarbonada é indicada entre parênteses, pela identificação do átomo de carbono mais próximo da carboxila implicado na respectiva insaturação. Os ácidos graxos também podem ser divididos em famílias ou séries, dependendo da localização da última ligação dupla em relação ao seu grupamento metílico terminal: família ômega-6 ( $\omega 6)$ representada pelo ácido linoléico e a família ômega-3 ( $\omega 3)$, pelo ácido $\alpha$-linolênico.

Esses dois tipos de ácidos graxos são chamados essenciais porque não podem ser sintetizados pelo organismo humano e devem ser fornecidos através da dieta. Após a ingestão, os ácidos graxos, uma vez absorvidos por células e tecidos, podem ser dessaturados e alongados a outros ácidos poliinsaturados de cadeia longa ${ }^{1}$. Os processos de alongação e dessaturação do ácidos linoléico e $\alpha$-linolênico ocorrem nos animais e, vagarosamente, nos homens originando diversos metabólitos, como representado na Figura 1.

$\mathrm{Na}$ atualidade, pesquisas têm enfatizado o importante papel dos metabólitos poliinsaturados, tais como ácido $\gamma$-linolênico (18:3 $(6,9,12))$ da família $\omega 6$, ácido eicosapentaenóico $(20: 5(5,8,11,14,17))$ e ácido docosaexaenóico $(22: 6(4,7,10,13,16,19))$ da família $\omega 3$, representados por EPA e DHA, respectivamente.

Os diversos dos ácidos graxos poliinsaturados (AGPI) no metabolismo de lipoproteínas, síntese de eicosanóides e funcionamento das plaquetas e das paredes dos vasos, os tornam de especial interes-

*e-mail: patcarvalho@saofrancisco.edu.br

\begin{tabular}{|c|c|c|}
\hline Família $\omega 6$ & & Família $\omega 3$ \\
\hline Linoléico (18:2) & & $\alpha$-Linolênico (18:3) \\
\hline$\Downarrow$ & $\Delta 6$ - dessaturase & $\Downarrow$ \\
\hline$\gamma$-Linolênico (18:3) & & Octadecatetraenóico (18:4) \\
\hline$\Downarrow$ & alongase & $\Downarrow$ \\
\hline Dihomo-- $\gamma$-linolênico(20:3) & & Eicosatetraenóico (20:4) \\
\hline$\Downarrow$ & $\Delta 5$ - dessaturase & $\Downarrow$ \\
\hline Araquidônico (20:4) & & Eicosapentaenóico (20:5) \\
\hline$\Downarrow$ & alongase & $\Downarrow$ \\
\hline Adrenico $(22: 4)$ & & Docosapentaenóico (22:5) \\
\hline$\Downarrow$ & $\Delta 4$ - dessaturase & $\Downarrow$ \\
\hline Docosapentaenóico (22:5) & & Docosahexaenóico (22:6) \\
\hline
\end{tabular}

Figura 1. Metabolismo de ácidos graxos essenciais pertencentes à família ômega-3 (w3) e ômega-6 (w6)

se em relação à prevenção e tratamento de diversas patologias cardiovasculares. A administração de óleos ricos em AGPI, ou seus concentrados, em humanos tem demonstrado efeitos benéficos na aterosclerose, trombose e arritmia ${ }^{2-4}$. Outros efeitos resultantes da ingestão dos AGPI foram observados em certos processos inflamatórios e alérgicos, como eczema atópico ${ }^{5}$, psoríase $^{6}$, asma ${ }^{7}$ e artrite reumatóide ${ }^{8}$. Também observaram-se efeitos na síndrome pré-menstrual $^{9}$, diabetes ${ }^{10}$ e de certos tipos de câncer ${ }^{11,12}$. Estudos enfatizam que estes ácidos afetam também as funções imunológicas, inibindo a proliferação de linfócitos, a produção de anticorpos e de citocinas pró-inflamatórias ${ }^{13,14}$.

Embora os AGPI possam ser sintetizados no corpo a partir da biotransformação dos ácidos graxos essenciais por reações de dessaturação e elongação, a ingestão direta dos metabólitos ativos tem se mostrado eficiente, principalmente para gestantes, idosos, lactantes, crianças prematuras ${ }^{15}$ e para indivíduos que apresentam insuficiência da enzima $\Delta 6$-dessaturase, decorrente de carências nutricionais ou do próprio envelhecimento ${ }^{16}$. O mercado mundial apresenta uma gama bastante ampla de suplementos alimentares (encapsulados) de AGPI $\omega 6$ e $\omega 3$ e de produtos nos quais estes áci- 
dos são incorporados, como leites e derivados, fórmulas lácteas infantis, biscoitos, pães, ovos, massas e sucos de frutas.

Em vista deste grande potencial, pesquisas voltadas a esta área têm relatado a aplicação de diferentes métodos físicos, químicos e enzimáticos visando a obtenção de concentrados de AGPI, a partir de substratos como óleos vegetais, animais e microbianos. Este trabalho tem por objetivo enumerar e discutir as principais publicações relacionadas à aplicação de lipases microbianas na obtenção de produtos com níveis aumentados em AGPI. Na primeira seção deste artigo, são relatados os estudos das reações catalisadas por lipases microbianas, que têm sido empregadas para obtenção dos concentrados de AGPI a partir de diferentes fontes e em variadas condições experimentais e, em seguida, uma comparação do processo enzimático com outros métodos físicos e químicos que permitem remover os componentes mais saturados do óleo.

\section{REAÇÕES CATALISADAS POR LIPASES PARA CONCENTRAÇÃO DE ÁCIDOS GRAXOS POLIINSATURADOS (AGPI)}

As lipases verdadeiras (triacilglicerol acilhidrolases E.C.3.1.1.3) são enzimas que catalisam a hidrólise total ou parcial de triacilglicerol (TAG) fornecendo diacilglicerol (DAG), monoacilglicerol (MAG), glicerol e ácidos graxos livres. Estas enzimas apresentam uma capacidade única de agir apenas na interface óleo/água. Esta definição exclui as enzimas que agem em ésteres solúveis em água (esterases) ou que hidrolizam outros lipídeos (acilidrolases, colesterolesterase, tioesterases e outras) $)^{17}$.

A partir dos trabalhos de Tsujisaka e colaboradores ${ }^{18}$ ficou comprovado ser possível reverter a hidrólise de TAG no sentido da reação de esterificação, pelo controle do teor de água no meio. Com base nisto, tornou-se possível também a utilização de lipases como biocatalisadores da interesterificação de óleos e gorduras ${ }^{19}$. Esta reação traz alterações na composição e distribuição dos ácidos graxos da molécula de TAG. Genericamente, a reação de interesterificação refere-se à troca de radicais acil entre um éster e um ácido (acidólise), um éster e um álcool (alcoólise), ou ainda de um éster e outro éster, na forma de glicerídeos ou de monoéster, reação intitulada por alguns autores de transesterificação. As reações catalisadas por lipases estão demonstradas esquematicamente na Figura 2.

Estas enzimas encontram-se largamente distribuídas na natureza em animais, vegetais e microrganismos. Apresentam peso molecular entre 40-50Kda com cerca de 300 resíduos de aminoácidos. São glicoproteínas nas quais a parte glicosilada hidrofóbica circunda o sítio ativo. As lipases provenientes de microrganismos são as mais utilizadas industrialmente porque além de apresentarem procedimentos mais simples de isolamento à partir do caldo fermentativo são, geralmente, mais estáveis e com propriedades bem mais diversificadas que as lipases de outras fontes. São, em sua maioria, extracelulares, favorecendo sua extração, isolamento e purificação ${ }^{20}$. O interesse em utilizar estes biocatalisadores na modificação estrutural de óleos e gorduras tem recebido considerável atenção devido, em especial, à sua especificidade em relação ao substrato ${ }^{21,22}$.

Segundo Macrae e Hammond ${ }^{23}$, as lipases microbianas têm sido classificadas em três grupos, em função de sua especificidade com relação ao substrato: Lipases 1,3 específicas - catalisam a liberação de ácidos graxos especificamente das posições $s n-1$ e $s n-3$ dos acilgliceróis. A hidrólise total pode ocorrer se a reação for por tempo prolongado, considerando-se que o 2- MAG ou 1,2 (2,3)- DAG formados, ambos quimicamente instáveis, podem ser isomerizados espontaneamente para as posições 1 ou 3. As lipases de Rhizopus delemar, Aspergillus niger e Mucor miehei pertencem a este grupo. A lipase pancreática é uma típica lipase $s n-1,3$ específica, e algumas lipases
Reação de Hidrólise

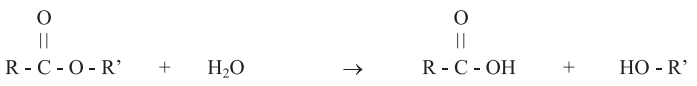

Reação de Esterificação

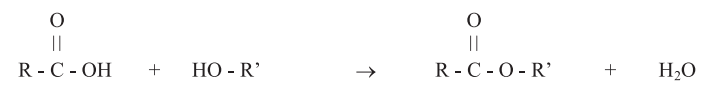

Reação de Interesterificação

Acidólise (reação de éster com ácido)

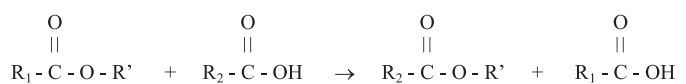

Alcoólise (reação de éster com álcool)

$$
\begin{aligned}
& \text { O } \\
& \mathrm{R}^{\prime}-\mathrm{C}-\mathrm{O}-\mathrm{R}_{1}+\mathrm{HO}-\mathrm{R}_{2} \rightarrow \mathrm{R} \text { - } \stackrel{\|}{\|}-\mathrm{O}-\mathrm{R}_{2}+\mathrm{HO}-\mathrm{R}_{1}
\end{aligned}
$$

Transesterificação (reação de éster com éster)

$$
\begin{array}{cccc}
\mathrm{O} & \mathrm{O} & \mathrm{O} & \mathrm{O} \\
\mathrm{R}_{1}-\mathrm{C}-\mathrm{O}-\mathrm{R}_{1}{ }^{\prime} & +\mathrm{R}_{2}-\mathrm{C}-\mathrm{O}-\mathrm{R}_{2}{ }^{\prime} \rightarrow \mathrm{R}_{1}-\mathrm{C}-\mathrm{O}-\mathrm{R}_{2}{ }^{\prime} & +\mathrm{R}_{2}-\mathrm{C}-\mathrm{O}-\mathrm{R}_{1},
\end{array}
$$

Figura 2. Representação esquemática das reações de hidrólise, esterificação e interesterificação catalisadas por lipases

vegetais (colza, mostarda e lupino) têm demonstrado possuir este tipo de especificidade. Lipases não específicas - catalisam a hidrólise de TAG para ácidos graxos livres e glicerol, de modo aleatório. Não mostram especificidade com relação à natureza do grupo acil ou à posição em que este está esterificado no glicerol. Exemplos são as lipases de Penicilium cyclopium, Corynebacterium acnes, Pseudomonas fluorescens, Staphylococcus aureus e Candida cylindracea. Lipases ácido graxo específicas - catalisam a hidrólise de tipos específicos de grupos acilas nas moléculas de TAG. Um representante típico deste grupo é a lipase de Geotrichum candidum que hidrolisa preferencialmente grupos acila de cadeia longa, que contenham dupla ligação cis na posição 9.

A reação enzimática de interesterificação tem algumas características em comum com as reações da química clássica correspondente, mas os princípios que governam a regioespecificidade são diferentes e justificam, em muitos casos, a utilização dos processos enzimáticos. Conforme a ilustração esquemática da Figura 3, os produtos resultantes da reação de interesterificação de uma mistura de TAGs (3b) ou entre TAG e ácidos graxos livres (3d), catalisada por lipases regioespecíficas para as posições 1 e 3 , permite que o processo seja dirigido para a formação de produtos com composição e propriedades peculiares. $\mathrm{O}$ mesmo pode ser constatado com o uso de lipase ácido graxo específica (3e). Em contrapartida, os produtos obtidos com o uso de lipases não regioespecíficas são similares àqueles obtidos por interesterificação química, onde ocorre uma redistribuição aleatória (randomização) dos ácidos graxos nos TAGs (3a e 3c) dificultando, em muitos casos, a obtenção de produtos com propriedades desejadas e com maior pureza ${ }^{24}$.

Embora as lipases tenham sido estudadas por muitos anos e possam ser produzidas em larga escala, estas enzimas têm sido de uso 


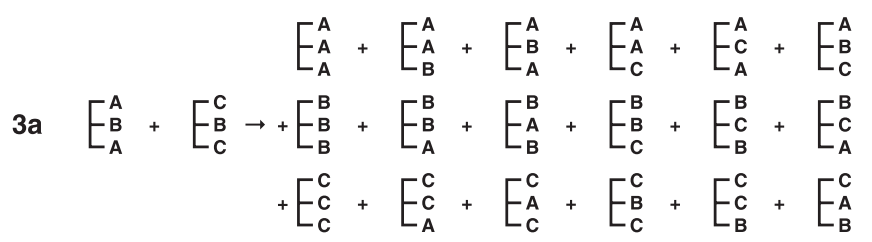

3b $E_{A}^{A}+E_{C}^{C} \rightarrow E_{A}^{A}+E_{C}^{A}+E_{C}^{C}$

$\begin{aligned} & E_{A}^{A}+E_{A}^{A}+E_{A}^{A}+E_{A}^{A}+E_{A}^{A}+E_{C}^{A} \\ 3 c \quad F_{A}^{A}+c \rightarrow+ & +E_{B}^{B}+E_{A}^{B}+E_{A}^{B}+E_{B}^{B}+E_{C}^{B}+E_{C}^{B} \\ & +E_{C}^{C}+E_{A}^{C}+E_{A}^{C}+E_{C}^{C}+E_{C}^{C}+E_{A}^{C} \\ & +A+B+C\end{aligned}$

$\begin{aligned} 3 d \quad E_{A}^{A}+c \rightarrow E_{A}^{A} & +E_{C}^{A}+E_{C}^{C} \\ & +A+c\end{aligned}$

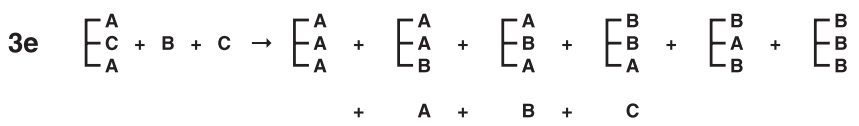

Figura 3. Produtos resultantes da reação de interesterificação química e enzimática de diferentes lipídeos (adaptada da ref. 24). 3a. reação de diferentes glicerídeos por catálise química ou com lipase não específca; $3 b$. reação de diferentes glicerídeos catalisada com lipase 1,3 específica; $3 c$. reação de glicerídeos e ácidos graxos livres por catálise química ou com lipase não específca; $3 d$. reação de glicerídeos e ácidos graxos livres catalisada com lipase 1,3 específca; 3 e. reação de glicerídeos e ácidos graxos livres catalisada por lipase com especificidade para ácido graxo $A$ e $B$

restrito da maioria das indústrias. Muitos estudos têm sido realizados com o intuito de otimizar os processos de modificação de óleos de gorduras catalisados por lipases; estes estudos incluem a imobilização da enzima, estudos cinéticos e desenvolvimento de bioreatores $^{19,25,26,56}$.

A aplicação de lipases com o objetivo de obter concentrados de AGPI de importância nutricional ou médica depende, em primeira instância, da capacidade de absorção destes ácidos pelos humanos. É conhecido que os ésteres de AGPI com álcoois primários apresentam absorção praticamente nula e podem resultar em metabólitos tóxicos. Por sua vez, os AGPI livres apresentam uma extensão de absorção significativa, mas possuem a desvantagem de sofrerem facilmente deterioração oxidativa. Em contrapartida, os AGPI esterificados ao glicerol na forma de acilgliceróis são melhores absorvidos, sendo esta forma a mais facilmente obtida pelas reações enzimáticas catalisadas pela lipase. Os estudos enzimáticos comentados a seguir estão relacionados à obtenção de produtos enriquecidos em AGPI na forma de mono, di e triacilgliceróis.

\section{HIDRÓLISE}

Os trabalhos pioneiros iniciaram-se com Bottino e colaborado$\mathrm{res}^{27}$, os quais demonstraram que os componentes EPA e DHA presentes em óleo de baleia resistiam à ação hidrolítica por lipase pancreática, resultando acilgliceróis parciais com níveis aumentados em
AGPI. Considerando que, em sua maioria, os AGPI dos óleos de peixe e outros óleos vegetais estão localizados na posição $s n-2$ do $\mathrm{TAG}^{28}$, os acilgliceróis resultantes da hidrólise parcial por lipases com especificidade posicional $s n-1$ e $s n-3$ perderão os ácidos saturados e monoinsaturados normalmente presentes nestas posições, resultando em aumento no conteúdo de AGPI e permitindo, por exemplo, a obtenção de $s n$-2 eicosapentaenoil glicerol e $s n$-2 docosaexaenoil glicerol após hidrólise com a lipase obtida de Mucor miehei (Lipozyme IM-20) ${ }^{29}$.

Lipases inespecíficas obtidas de Candida cylindracea $a^{26,30-33} \mathrm{e}$ Pseudomonas fluorescens ${ }^{34}$ também podem ser eficientes para enriquecer os AGPI nos produtos da hidrólise. A atuação da lipase inespecífica de Candida cylindracea chegou a triplicar a concentração original de DHA após $60 \%$ de hidrólise do óleo de peixe. O autor atribuiu este fato à hidrólise preferencial de alguns ácidos saturados e poliinsaturados e à possível "discriminação" desta enzima em relação ao DHA, baseado na especificidade quanto ao tipo de ácido graxo pela enzima ${ }^{31}$, ou, conforme postulado posteriormente, à especificidade pela molécula inteira de TAG que contenha o DHA ${ }^{32}$. Esta lipase mostrou ser mais eficiente no enriquecimento de DHA do óleo de atum quando comparada com outras lipases inespecíficas obtidas de Aspergillus niger, Chromobacterium viscosum, Pseudomonas $s p^{31}$ e com lipase obtida de Geotrichum candidum (especificidade de ácido graxo) e de Rhizopus delemar (sn 1,3 específica) ${ }^{33}$. Níveis superiores a 50\% de AGPI $\omega 3$ em óleo marinho foram obtidos utilizando lipase imobilizada de Candida cylindracea ${ }^{26,35}$.

Outros AGPI importantes, como o ácido $\gamma$-linolênico e o ácido araquidônico, têm recebido considerável atenção. Hills e colaboradores deram início a uma série de estudos visando concentrar o ácido $\gamma$-linolênico obtido de fontes vegetais. Os autores descreveram a hidrólise de óleo de prímula (Oenothera biennis L. contendo 9,5\% de ácido $\gamma$-linolênico) catalisada por lipase vegetal (Brassica napus L. $)^{36}$. Nas frações MAG e DAG, o conteúdo de ácido $\gamma$-linolênico resultante foi de 45 e $28 \%$, respectivamente, demonstrando resistência do ácido $\gamma$-linolênico à atuação enzimática. Syed Rahmatullah e colaboradores $^{37}$ relataram posteriormente que lipase de Candida cylindracea também se mostrou eficiente para o enriquecimento de ácido $\gamma$-linolênico após hidrólise parcial do óleo de semente de prímula (Oenothera biennis L.) e borragem (Borago officinallis L.).

\section{ESTERIFICAÇÃO}

A reação de esterificação entre álcoois poliídricos e ácidos graxos livres é a reação inversa da hidrólise do acilglicerol correspondente. A relação entre as velocidades da reação direta (hidrólise) e da reação inversa (esterificação) é usualmente controlada pelo conteúdo de água do meio de reação. Um grau elevado de incorporação dos AGPI ao glicerol foi relatado por Osada e colaboradores ${ }^{38}$ com as lipases de Chromobacterium viscosum (89-90\%) e Candida cylindracea $(70 \%)$, sugerindo ser este um método praticável para produção de acilgliceróis ricos em AGPI. Posteriormente, foi relatado que a lipase obtida de Mucor miehei também era capaz de incorporar os AGPI ao glicerol ${ }^{39}$.

Li Zu Yi e Ward ${ }^{40}$ relataram a obtenção de acilgliceróis contendo aproximadamente $70 \%$ de AGPI (EPA + DHA), respectivamente, após 24 h de reação de esterificação em mistura de reação contendo 2,0 $\mathrm{g}$ de glicerol, 0,4 $\mathrm{g}$ de AGPI, 3,0 mL de hexano, 5\% de água e $5.000 \mathrm{U}$ da lipase de Pseudomonas sp. Os mesmos autores relataram, posteriormente, um método enzimático para produção de $80 \%$ de MAG contendo $76,2 \%$ de AGPI por lipase microbiana obtida de Mucor miehei em meio com solvente orgânico ${ }^{41}$.

O grau de esterificação é altamente dependente das condições de reação, em especial do conteúdo de água reacional para que a hidrólise 
seja evitada. Medina e colaboradores ${ }^{42}$ relataram a obtenção de $93,5 \%$ de TAG contendo $25,7 \%$ de EPA e $44,7 \%$ de DHA usando como substrato o óleo de peixe e a obtenção de $89,3 \%$ de TAG contendo 45,6\% de EPA e 43,4\% de ácido araquidônico usando como substrato a microalga Phaeodactylum cruentum, em meio reacional orgânico, isento de água. A adição de solventes orgânicos no meio tem sido usada como forma de impedir a hidrólise e aumentar a solubilidade de substratos hidrofóbicos, tornando viável a reação de esterificação.

A lipase obtida de Geotrichum candidum foi relatada como sendo capaz de esterificar os ácidos oléico (18:1 (9)), linoléico (18:2 $(9,12))$ e $\alpha$-linolênico $(18: 3(9,12,15))$ com butanol, enquanto os ácidos graxos de cadeia carbônica maior que $\mathrm{C} 18$, como o ácido erúcico (C22:1 (6)), ou ácidos insaturados como ácido $\gamma$-linolênico $(18: 3(6,9,12))$ e petroselênico $(18: 3(6))$, mostraram uma taxa de esterificação nula ${ }^{43}$. Esta possível "discriminação" pode ser aproveitada para a obtenção de concentrados de AGPI na fração não esterificada. A concentração de ácido $\gamma$-linolênico de $24,9 \%$ em relação aos ácidos graxos totais do óleo de borragem (Borago officinalis) subiu para $71,8 \%$ na fração ácidos graxos não esterificados com butanol após 48 h de reação ${ }^{44}$. A reação preferencial de esterificação do butanol com os ácidos palmítico, esteárico, oléico e linoléico obtidos do óleo de Mucor sp foi utilizada para obter concentrados de ácido $\gamma$-linolênico na fração ácidos graxos não esterificadas após catálise por lipase de Geotrichum $\mathrm{sp}^{45}$.

O aproveitamento dos constituintes oleosos do caldo (efluentes) da indústria de enlatados de sardinha, visando o enriquecimento do EPA e DHA neste óleo, após hidrólise com a lipase de Candida rugosa, seguida da esterificação seletiva com a lipase de Lipozyme (Rhizomucor miehei) foi objeto de estudo de Schmitt-Rozieres $e$ colaboradores $^{46,47}$.

\section{INTERESTERIFICAÇÃO}

Na reação de interesterificação, o TAG reage com um ácido graxo, um álcool ou outro éster, resultando em rearranjo na distribuição dos ácidos graxos do TAG formando um novo TAG, com propriedades químicas e físicas diversas. O rearranjo é o resultado de reações concorrentes de hidrólise e esterificação. Em geral, sob condições nas quais a quantidade de água no sistema é restrita, a hidrólise de gordura pode ser minimizada de tal forma que a interesterificação seja a reação dominante ${ }^{48}$. A reação de interesterificação tem demonstrado aumentar significativamente os níveis de AGPI nos acilgliceróis resultantes. A obtenção de acilgliceróis contendo $70 \%$ de AGPI $\omega 3$, em especial DHA (48\%) e EPA (12\%), foi realizada via interesterificação de óleo de fígado de bacalhau e concentrados de AGPI livres, ou seus ésteres, catalisada por lipase imobilizada de Mucor $m i e h i^{49}$. A mesma enzima foi usada para a obtenção de óleo de sardinha contendo 25\% de EPA e $40 \%$ de DHA após acidólise entre o óleo de sardinha e AGPI livres na ausência de solvente ${ }^{50}$. Na seleção de lipases microbianas utilizadas para aumentar os níveis de AGPI do óleo de sardinha após reação de acidólise, a lipase de Pseudomonas sp apresentou a maior capacidade entre as lipases testadas, tendo o conteúdo total destes ácidos graxos atingido $44 \%$ nos acilgliceróis, comparado com $29 \%$ do óleo de sardinha original ${ }^{51}$.

A reação de interesterificação também tem sido utilizada para tornar os óleos vegetais mais ricos em AGPI de interesse nutricional. $\mathrm{Li} \mathrm{Zu} \mathrm{Yi} \mathrm{e}$ Ward $^{52}$ relataram a incorporação de aproximadamente $20 \%$ (em relação aos ácidos graxos totais) de AGPI nos óleos de milho, girassol, amendoim, oliva e soja usando lipase imobilizada obtida de Mucor miehei e de Pseudomonas sp, a qual mostrou alta atividade catalítica e permitiu o uso repetido da enzima imobilizada sem perda significativa na sua atividade ${ }^{53}$.
A incorporação de AGPI $\omega 3$ em óleo de soja após transesterificação catalisada por lipase de Candida cylindracea formou TAGs com $34,7 \%$ e 32,9\% de EPA e DHA, respectivamente ${ }^{54}$. Akoh e colaboradores ${ }^{55}$ descreveram a reação de transesterificação entre óleo de prímula (ácido $\gamma$-linolênico) e éster etílico de EPA como doadores de grupo acil, catalisada por lipase imobilizada de Candida antarctica. A incorporação de EPA foi de 34\%, enquanto os níveis de ácido $\gamma$-linolênico não sofreram mudanças significativas, o que resultou em uma modificação favorável na taxa de $\omega 3 / \omega 6$ de 0,01 para 0,60 após reação de transesterificação. Alguns estudos têm utilizado com sucesso a lipase obtida de Candida antarctica na catálise da reação de transesterificação de diferentes ésteres (acilgliceróis e ésteres com álcool primário) na obtenção de óleos enriquecidos com AGPI para propósitos nutracêuticos ${ }^{56}$.

A Tabela 1 traz um sumário das principais referências disponíveis, a partir da década de 90 , sobre a obtenção de concentrados de AGPI usando lipases.

\section{MÉTODOS FÍSICOS E QUÍMICOS PARA CONCENTRAÇÃO DE ÁCIDOS GRAXOS POLIINSATURADOS (AGPI)}

O processo de interesterificação química é realizado através do aquecimento de misturas de óleos e ácidos graxos na presença de catalisadores como o sódio metálico, metilato ou etilato de sódio e outros alquilatos de metais alcalinos ( $\mathrm{Na}, \mathrm{K}$ e suas ligas). Durante este processo, a mistura é aquecida e os ácidos graxos trocam de posição na molécula do TAG. A variabilidade na qualidade do catalisador, a necessidade de um período de indução e perdas de óleo pela formação de sabões de ésteres metílicos são algumas das desvantagens da interesterificação química. Além disto, na interesterificação química ocorre a redistribuição aleatória dos ácidos graxos nos TAGs (randomização), dificultando que óleos e gorduras obtidos demonstrem propriedades desejáveis ${ }^{23}$.

Os estudos relatados na literatura para extração, concentração e purificação dos AGPI incluem métodos físicos e químicos, tais como complexação com uréia ${ }^{64,65}$, resfriamento por solvente ${ }^{60}$, cristalização fracionada à baixa temperatura ${ }^{67}$, extração com fluido super crítico $^{68} \mathrm{e}$ extração baseada na solubilidade dos sais de ácidos graxos em diferentes solventes orgânicos ${ }^{69}$. A separação seletiva de AGPI por outros métodos como cromatografia líquida de alta eficiência em fase reversa ${ }^{70}$, coluna de troca iônica usando zeolita ${ }^{71}$, e o método de Corey ${ }^{72,73}$ constituíram outras alternativas relatadas em nível experimental.

O método de fracionamento com complexação (inclusão) com uréia baseia-se na separação pelo grau de insaturação, sendo que os ácidos graxos mais insaturados são menos incluídos nos adutos de uréia. Haagsma e colaboradores ${ }^{64}$ relataram a preparação de um concentrado de ácidos graxos $\omega 3$ via complexação com uréia contendo $85 \%$ de AGPI na forma livre.

O método de extração em fluido super crítico com dióxido de carbono tem recebido destaque nas publicações mais recentes. Esta técnica tem sido apontada como de larga aplicação em alimentos e produtos farmacêuticos, que envolvem o manuseio de compostos bioativos e termolábeis. Bons resultados foram obtidos usando ésteres metílicos ou etílicos de AGPI, apesar do alto custo operacional e do pequeno interesse comercial nestes ésteres ${ }^{75}$.

O procedimento usando duas etapas, inclusão em uréia seguida de cromatografia líquida de alta eficiência (HPLC) preparativa em coluna de fase reversa $\mathrm{C} 18$, permitiu a obtenção de concentrados de EPA e DHA a partir do óleo de fígado de bacalhau e da microalga Isochrysis galbana. A associação dos métodos, embora de alto custo, mostrou-se eficiente, resultando em um concentrado contendo 95\% de AGPI livres ${ }^{74}$. 
Tabela 1. Enriquecimento de AGPI por reações de hidrólise, esterificação e interesterificação usando lipases de diferentes fontes

\begin{tabular}{|c|c|c|c|c|}
\hline Enzima & Estado & Reação catalisada & AGPI enriquecido & Referência \\
\hline $\mathrm{CC}$ e $\mathrm{AN}$ & Livre & Hidrólise & EPA/DHA/ DPA & 30 \\
\hline $\mathrm{CC}$ e $\mathrm{CV}$ & Livre & Esterificação & EPA/DHA/GLA & 38 \\
\hline MM e LN & Imobilizada & Esterificação & DHA/GLA & 57 \\
\hline $\mathrm{MM}$ & Livre & Hidrólise & GLA & 58 \\
\hline MM & Livre & Esterificação & GLA & 58 \\
\hline $\mathrm{CC}$ & Livre & Hidrólise & DHA & 31 \\
\hline $\mathrm{MM}$ e $\mathrm{CV}$ & Livre & Esterificação & EPA/DHA & 39 \\
\hline MM & Imobilizada & Interesterificação & EPA/DHA & 53 \\
\hline $\mathrm{CC}$ & Imobilizada & Hidrólise & GLA & 37 \\
\hline MM & Imobilizada & Esterificação & GLA & 59 \\
\hline GC e RD & Livre & Hidrólise & EPA/DHA & 33 \\
\hline $\mathrm{CV}$ & Imobilizada & Esterificação & DHA & 60 \\
\hline GC & Imobilizada & Esterificação & GLA & 44 \\
\hline $\mathrm{GC}$ & Livre & Hidrólise & EPA/DHA & 61 \\
\hline $\mathrm{CC}$ & Livre & Hidrólise & $\mathrm{AA}$ & 62 \\
\hline $\mathrm{CA}$ & Imobilizada & Interesterificação & GLA/EPA & 55 \\
\hline RD & Livre & Esterificação & DHA & 63 \\
\hline $\mathrm{CC}$ & Imobilizada & Hidrólise & EPA/DHA/DPA & 35 \\
\hline $\mathrm{CC}$ & Imobilizada & Hidrólise & EPA/DHA & 26 \\
\hline MM & Imobilizada & Hidrólise & EPA/DHA & 29 \\
\hline $\mathrm{CA}$ & Imobilizada & Esterificação & EPA/DHA & 42 \\
\hline MM & Imobilizada & Esterificação & EPA/DHA & 46 \\
\hline CC e MM & Imobilizada & Esterificação & EPA/DHA & 47 \\
\hline $\mathrm{CA}$ & Imobilizada & Interesterificação & EPA/DHA & 56 \\
\hline
\end{tabular}

MM: Mucor miehei (Lipozyme); LN: Lipase vegetal de nabo; CC: Candida cylindracea (rugosa); CA: Candida antarctica; AN: Aspergillus niger; CV: Chromobacterium viscosum; GC: Geotrichum candidum; EPA: ácido eicosapentaenóico; DHA: ácido docosahexaenóico; DPA: ácido docosapentaenóico; GLA: ácido $\gamma$-linolênico; AA: ácido araquidônico; RD: Rhizopus delemar; PF: Pseudomonas fluorescens

\section{CONCLUSÕES}

As lipases têm um importante papel devido ao fato de serem disponíveis comercialmente, obtidas a partir de diferentes fontes, serem seletivas em relação aos seus substratos e também catalisadores muito eficientes mesmo sob condições brandas de operação, nas quais os grupos acila não sofrem alterações indesejáveis. De modo especial, a reação de interesterificação delinea possibilidades muito interessantes. A ação da lipase nas posições 1 e 3 do TAG possibilita a obtenção de gorduras com características especiais promovendo uma troca de grupamentos acil apenas nestas posições, o que não pode ser alcançado por via química. Além disto, a interesterificação química consome muita energia (alta temperatura e pressão), deixa resíduos tóxicos e os ácidos graxos obtidos são escuros e impuros, devido às reações secundárias que ocorrem no processo e, desse modo, para se chegar aos produtos finais são necessárias etapas adicionais de purificação.

Os processos físicos e químicos para obtenção de concentrados de AGPI são geralmente de alto custo operacional e têm demonstrado ser eficientes somente para obtenção de ésteres de ácidos ou AGPI na forma livre, de baixo interesse farmacêutico.

A característica de instabilidade em meio orgânico, a faixa limitada de substrato específico e o alto custo apontados como os problemas mais sérios para o uso das lipases têm sido superados graças aos novos desenvolvimentos tecnológicos. A utilização de lipases tem aberto um novo caminho para a biotecnologia industrial de óleos e gorduras, possibilitando a obtenção de concentrados de AGPI na forma de glicerídeos (MAG, DAG e TAG) com boas perspectivas de aplicação.

\section{AGRADECIMENTOS}

Os autores agradecem à FAPESP e ao CNPq pelo apoio financeiro.

\section{REFERÊNCIAS}

1. Lands, W. E. W.; Nutr. Rev. 1986, 44, 189.

2. Dyeberg, J.; Bang, H. O.; Stoffersen, E; Moncada, S.; Vane, J. R.; Lancet 1978, 2, 117.

3. Drevon, A. C.; Nutr. Rev. 1992, 50, 38.

4. Horrobin, D. F.; Prog. Lipid Res. 1992, 31, 163.

5. Manku, M. S.; Horrobin, D. F.; Morse, N. L.; Wright, S.; Burton, J. L.; Br. J. Dermatol. 1984, 110, 643.

6. Barlow, S.; Infofish Mark. Dig. 1987, 1, 38.

7. Masuev, K. A.; Ter. Arkh. 1997, 69, 31.

8. Kremer, J. M.; Jubiz, W.; Michalek, A.; Ann. Int. Med. 1987, 106, 497.

9. Harel, Z.; Biro, F. M.; Kottenhahn, R. K.; Rosenthal, S. L.; Am. J. Obstet. Gynecol. 1996, 174, 1335.

10. Keen, H.; Payan, J.; Allawi, J.; Walker, J.; Jamal,G.A.; Weir, A.I.; Henderson, L. M.; Bissessar, E. A.; Watkins, P. J.; Sampson, M.; Gale, E. A. M.; Scarpello, J.; Boddie, H. G.; Hardy, K. J.; Thomas, P. K.; Misra, P.; Halonen, J. P.; Diabetes Care 1993, 16, 8.

11. Bagga, D.; Capone, S.; Wang, H. J.; Heber, D.; Lill, M.; Chap, L; Glaspy, J. A.; J. Natl. Cancer Inst. 1997, 89, 1123.

12. Whitehouse, A. S.; Smith, H. J.; Tisdale, M. J.; Cancer Res. 2001, 61, 3604.

13. De Pablo, M. A.; Puertollano, M. A.; De Cienfuegos, G. A.; FEMS Immunol. Med. Microbiol. 2000, 29, 323.

14. Calder, P. C.; Nutr. Res. 2001, 21, 309.

15. Innis, S. M.; Dev. Neurosci. 2000, 22, 474.

16. Kinsella, J. E.; Adv. Food Nutr. Res. 1991, 35, 1.

17. Brockman, H. L.; Momsen, W. E.; Tsujita, T.; J. Am. Oil Chem. Soc. 1988, 65, 891. 
18. Tsujisaka, Y.; Okumura, S.; Iwai, M.; Biochim. Biophys. Acta 1977, 489, 415.

19. Quinlan, P.; Moore, S.; Inform. 1993, 4, 580.

20. Borgston, B.; Brockman, H. L.; Lipases, $4^{\mathrm{a}}$ ed., Elsevier: Amsterdan, 1984.

21. Gunstone, F. D.; J. Sci. Food Agric. 1999, 79, 1535.

22. Xu, X. B.; Eur. J. Lipid Sci. Technol. 2000, 102, 287.

23. Macrae, A. R.; Hammond, R. C.; Biotechnol. Genet. Eng. Rev. 1985, 3, 193.

24. Macrae, A. R.; J. Am. Oil Chem. Soc. 1983, 60, 243.

25. Valenzuella, A.; Nieto, S.; Grasas y Aceites 1994, 45, 337.

26. Rice, K. E.; Watkins, J.; Hill, C. G.; Biotechnol Bioeng. 1999, 63, 33

27. Bottino, N. R.; Vandenburg, G. A.; Reiser, R.; Lipids 1967, 2, 489.

28. Brockerhoff, H.; Hoyle, H. J. ; Wang, P. C.; Litchfield, C.; Lipids 1969, 3 , 24.

29. Nieto, S.; Gutierrez, J.; Sanhueza, J.; Valenzuela, A.; Grasas y Aceites 1999, $50,111$.

30. Hoshino, T.; Yamane, T.; Shimizu, S.; Agric. Biol. Chem. 1990, 54, 1459.

31. Tanaka, Y.; Hirano, J.; Funada T.; J. Am. Oil Chem. Soc. 1992, 69, 1210.

32. Tanaka, Y.; Funada T.; Hirano, J.; Hashizume, R.; J. Am. Oil Chem. Soc. 1993, 70, 1031.

33. Shimada, Y.; Maruyama, K.; Okazaki, S.; Nakamura, M.; Sugihara, A.; Tominaga, Y.; J. Am. Oil Chem. Soc. 1994, 71, 951.

34. Rakshit, S. K.; Vasuhi, R.; Kosugi, Y.; Bioprocess Biosyst. Eng. 2000, 23, 251.

35. Wanasundara, U. N.; Shahidi, F.; J. Am. Oil Chem. Soc. 1998, 75, 1767.

36. Hills, M. J.; Kiewitt, I.; Murherjee, K. D.; Biotechnol. Lett. 1989, 9, 629.

37. Syed Rahmatullah, M. S. K.; Shukla, V. K. S.; Mukherjee, K. D.; J. Am. Oil Chem. Soc. 1994, 71, 569.

38. Osada, K.; Takahashi, K.; Hatano, M.; J. Am. Oil Chem. Soc. 1990, 67, 921.

39. Lie, E.; Molin, G.; Int. J. Food Sci. Technol. 1992, 27, 73.

40. Li Zu Yi; Ward, O. P.; J. Am. Oil Chem. Soc. 1993, 70, 745

41. Li Zu Yi; Ward, O. P.; J. Ind. Microbiol. 1994, 13, 49.

42. Medina, A. R.; Cerdan, L. E.; Giménez, A. G.; Paes, B. C.; Gonzales, M. J. I.; Grima, E. M.; J. Biotechnol. 1999, 70, 379.

43. Sonnet, P. E; Foglia, T. A.; Baillargeon, W.; J. Am. Oil Chem. Soc. 1993, $70,1043$.

44. Foglia, T. A.; Sonnet, P. E.; J. Am. Oil Chem. Soc.1995, 72, 417.

45. Carvalho, P. O.; Pastore, G. M.; Food Biotecnol. 1998, 12, 57.

46. Schmitt-Rozieres M.; Guilherm, M.C.; Phan T.L.R.; Corneau, L.C.; Biocatal. Biotransform. 2000, 18, 355 .

47. Schmitt-Rozieres M.; Deyris, V.; Corneau, L. C.; J. Am. Oil Chem. Soc. 2000, 77, 329 .
48. Goldberg, M.;Thomas, D.; Legoy, M.-D.; Eur. J. Biochem. 1990, 190, 603. 49. Haraldsson, G. G.; Höskuldsson, P. A.; Tetrahedron Lett. 1989, 30, 1671.

50. Yamane, T.; Suzuki, T.; Sahashi, Y.; Vikersveen, L.; Hoshino, T.; J. Am. Oil Chem. Soc. 1992, 69, 1104.

51. Adachi, S.; Okumura, K.; Ota, Y.; Mankura, M.; J. Ferment. Bioeng. 1993, 75,259

52. Li Zu Yi; Ward, O. P.; Biotechnol. Lett. 1993, 15, 185.

53. Li Zu Yi; Ward, O. P.; Biotechnol. Lett. 1993, 15, 393.

54. Huang, K. H.; Akoh, C. C.; J. Am. Oil Chem. Soc. 1994, 71, 1277.

55. Akoh, C. C.; Jennings, B. H.; Lillard, D. A.; J. Am. Oil Chem. Soc. 1996, $73,1059$.

56. Osorio, N. M.; Ferreira-Dias, S. ; Gusmao, J. H.; Da Fonseca, M. M. R.; J. Mol. Catal. B: Enzym. 2001, 11, 677.

57. Hills, M. J.; Kiewitt, I.; Murherjee, K. D.; J. Am. Oil Chem. Soc. 1990, 67,561

58. Mukherjee, K. D.; Kiewitt, I.; Appl. Microbiol. Biotechnol. 1991, 35, 579.

59. Syed Rahmatullah, M. S. K.; Shukla, V. K. S.; Mukherjee, K. D.; J. Am. Oil Chem. Soc. 1994, 71, 563.

60. Tanaka, Y.; Hirano, J.; Funada T.; J. Am. Oil Chem. Soc. 1994, 71, 331.

61. Shimada, Y.; Maruyama, K.; Nakamura, M.; Nakayama, S.; Sugihara, A.; Tominaga, Y.; J. Am. Oil Chem. Soc. 1995, 72, 1577.

62. Shimada, Y.; Sugihara, A.; Maruyama, K.; Nagao, T.; Nakamura, M.; Nakano, H.; Tominaga, Y.; J. Am. Oil Chem. Soc. 1995, 72, 1323.

63. Shimada, Y.; Sugihara, A.; Nakano, H.; Kuramoto, T.; Nagao, T.; Gemba, M.; Tominaga, Y.; J. Am. Oil Chem. Soc. 1997, 74, 97.

64. Haagsma, N.; Gent, C. M.; Luten, J. B.; Jong, R. W.; Doorn, E.; J. Am. Oil Chem. Soc. 1982, 59, 117.

65. Ackman, R. G.; Ratnayake, W. M. N.; Olsson, B.; J. Am. Oil Chem. Soc. 1988, 65, 136

66. Yokochi, T.; Usita, M. T.; Kamisaka, Y.; Nakahara, T.; Sukuki, O.; J. Am. Oil Chem. Soc. 1990, 67, 846.

67. Moffat, C. F.; Mcgill, A. S.; Hardy, R.; Anderson, R. S.; J. Am. Oil Chem. Soc. 1993, 70, 133.

68. Nilsson, W. B.; Gauglitz, Jr. E. J.; Hudson, J. K.; J. Am. Oil Chem. Soc. 1991, 68, 87.

69. Chen, T. C.; Ju, Y.H.; J. Am. Oil Chem. Soc. 2000, 77, 425.

70. Hidajat, K.; Ching, C. B.; Rao, M. S.; J. Chromat. 1995, 702, 215.

71. Arai, M.; Fukuda, H.; Morikawa, H.; J. Ferment Technol. 1987, 65, 569.

72. Corey, E. J.; Wright, S. W.; J. Org. Chem. 1987, 53, 5980.

73. Wright, S. W.; Kuo, E. Y.; Corey, E. J.; J. Org. Chem. 1987, 52, 4399.

74. Medina, A. R.; Giménez, A. G.; Camacho, F. G.; Pérez, J. A. S.; Grima, E. M.; Gómez, A. C.; J. Am. Oil Chem. Soc. 1995, 72, 575.

75. Shadidi, F.; Wanasundara, U. N.; Trends Food Sci. Technol. 1998, 9, 230 